\title{
Banco de sementes em cerrado sensu stricto sob queimada e sistemas de cultivo
}

\author{
Fernanda Satie lkeda ${ }^{(1)}$, Danielle Mitja(2), Lourival Vilela(1) e José Carlos Sousa Silva ${ }^{(1)}$
}

\begin{abstract}
(1)Embrapa Cerrados, Caixa Postal 08223, CEP 73310-970 Planaltina, DF. E-mail: fernanda.satie.ikeda@gmail.com, Ivilela@cpac.embrapa.br, jcarlos@cpac.embrapa.br (2)Institut de Recherche pour le Développement (IRD), US Espace 140 et UMR 137 - Domaine de Lavalette - Centre IRD de Montpellier - 911, Avenue Agropolis, BP 64501 - F- 34394 Montpellier, Cedex 5, França. E-mail: danielle.mitja@ird.fr
\end{abstract}

\begin{abstract}
Resumo - O objetivo deste trabalho foi avaliar o efeito de queimada e de sistemas de cultivo sobre o banco de sementes em solo de Cerrado. Foi realizada amostragem em área sob cerrado sensu stricto queimada e não queimada, assim como em área de pastagem e lavoura com preparo convencional do solo. Não houve redução no banco de sementes, embora tenha havido menor número de espécies e de famílias, e dos índices de diversidade, com a queimada. Houve maior índice de valor de importância (IVI) e número de espécies nativas, na área não queimada, e de espécies invasoras na área queimada. Na área de lavoura, houve maior densidade de sementes do que na área de pastagem e de cerrado. As espécies com o maior IVI nas áreas foram aquelas consideradas invasoras e forrageiras. A área de lavoura apresentou menores IVI de espécies nativas, índices de diversidade (Shannon e Simpson) e percentagem de espécies nativas do que as áreas de pastagem e cerrado. O cultivo com lavoura causa maior perturbação nas áreas do que a queimada.
\end{abstract}

Termos para indexação: diversidade, fitossociologia, lavoura, pastagem, planta daninha.

\section{Seedbanks in cerrado sensu stricto under burning and cultivation system}

\begin{abstract}
The objective of this work was to evaluate the burning and cultivation effects on soil seedbanks originally under cerrado vegetation. Two areas under cerrado sensu stricto were sampled, one burned and the other one not burned, as well as areas under tilled crop and pasture. There was no reduction on seedbank, although lower number of species and families and diversity index (Shannon and Simpson) with burning were observed. There was also greater importance value index (IVI) and number of native species, in the non burned area, and of weeds in the burned one. It was observed that the crop area had higher seed density than the pasture and cerrado areas. The species with the highest IVI in the areas were those considered as weeds and forages. The crop area presented lower IVI of native species than the pasture and cerrado areas; besides, it presented diversity indexes (Shannon and Simpson) and native species percentage lower than pasture and cerrado. The cultivation with crop cause higher disturbance in the areas than burning.
\end{abstract}

Index terms: diversity, phytosociology, crop, pasture, weed.

\section{Introdução}

O bioma Cerrado tem apresentado maior pressão antrópica, decorrente principalmente da atividade pecuária (Sano et al., 2002). No Brasil, a área do bioma Cerrado corresponde a aproximadamente 1,8 milhões de $\mathrm{km}^{2}$ (Aguiar et al., 2004), e 13\% são ocupados com pastagens nativas, $23 \%$ com pastagens cultivadas, $5 \%$ com culturas agrícolas, $18 \%$ com outros tipos de uso, e $41 \%$ correspondem às áreas não cultivadas (Sano et al., 2001). O efeito dessa antropização, sobre as espécies encontradas em áreas originalmente sob vegetação de Cerrado, pode ser analisado sob o ponto de vista ecológico e o de produção agropecuária. No primeiro caso, esse efeito está relacionado à perda da riqueza de espécies nativas desse bioma (Felfili \& Silva Júnior, 2001). No segundo, as espécies consideradas nativas de cerrado que se mantiveram nas áreas cultivadas podem ser tidas como invasoras que geralmente comprometem a produtividade agropecuária (Pott et al., 2006). Nesses casos, o estudo do banco de sementes pode ser importante, para a melhor compreensão da dinâmica de espécies nativas de cerrado nas áreas cultivadas, assim como a relação dessas espécies com aquelas consideradas invasoras verdadeiras.

Paralelamente ao aumento da antropização, foi intensificada a ocorrência de queimadas em áreas de 
cerrado nativo. Assim, queimadas acidentais ou criminosas são freqüentes na época de seca, na Região do Cerrado, e podem também ser praticadas em decorrência das atividades agropecuárias (Mistry, 1998). Ademais, a queimada é, em princípio, um dos principais agentes de modificação da estrutura e composição das comunidades vegetais em ecossistemas savânicos (Medeiros \& Miranda, 2005), pois proporciona oportunidade de competição das espécies invasoras com a regeneração das espécies-clímax (Felfili \& Silva Júnior, 2001). Além disso, a queimada também afeta a dinâmica das espécies vegetais, assim como o banco de sementes (Heringer \& Jacques, 2001). Entretanto, praticamente não há trabalhos que tenham avaliado o banco de sementes em áreas de cerrado nativo, ou mesmo tenham relacionado o efeito da queimada e de sistemas de cultivo sobre o banco de sementes dessas áreas. Na avaliação realizada por Sassaki et al. (1999), determinou-se a densidade de sementes em área de cerrado sem queimada, no entanto não se identificaram as espécies. Para isso, faz-se, geralmente, a avaliação do banco de sementes pelo método de emergência de plântulas em casa de vegetação (Ball \& Miller, 1989). Com isto podese calcular o índice de valor de importância (Moonen \& Bàrberi, 2004; Sosnoskie et al., 2006) e os índices de diversidade (Murphy et al., 2006).

Este trabalho teve como objetivo avaliar a influência de sistemas de cultivo e de queimada sobre o banco de sementes, em solo de cerrado sensu stricto, em Planaltina, DF.

\section{Material e Métodos}

Este trabalho foi realizado em uma área experimental da Embrapa Cerrados, em Planaltina, DF, a 1539'84"S e $47^{\circ} 44^{\prime} 41^{\prime \prime} \mathrm{W}$, em altitude aproximada de $1.217 \mathrm{~m}$. O clima no local foi classificado como Aw (Köppen), com duas estações bem definidas (seca e chuvosa) e a possibilidade de ocorrência de períodos de estiagem durante a estação chuvosa. A vegetação de cerrado sensu stricto, em solo classificado como Latossolo Vermelho, com textura argilosa, foi desmatada em 1991, ano de instalação do experimento. Parte da vegetação da área experimental foi queimada acidentalmente no dia 29 de setembro de 2004.

Para a avaliação do efeito de sistemas de cultivo sobre o banco de sementes de espécies nativas de cerrado, coletaram-se amostras em uma área de pastagem contínua (40x50 m), outra de lavoura contínua (20x50 m), e em uma área com cerrado sensu stricto $(40 \times 200 \mathrm{~m})$ em que não ocorreu nenhuma queimada de 1991 a 2004. $\mathrm{Na}$ avaliação do efeito de queimada, foi efetuada também uma coleta na área de cerrado sensu stricto onde houve apenas a queimada de setembro de 2004 registrada em seu histórico.

O histórico das áreas com a seqüência de culturas e o total de calcário e de fertilizantes aplicados, em cada sistema de cultivo, encontram-se descritos na Tabela 1.

$\mathrm{Na}$ área de lavoura, o preparo convencional do solo foi efetuado mediante uma gradagem pesada, após a colheita, e uma gradagem pesada seguida por uma gradagem de nivelamento, antes da semeadura. Os tratos culturais foram aplicados de acordo com as recomendações de cada cultura. Nos anos de cultivo da soja, foram aplicados os herbicidas trifluralin e imazaquin em pré-emergência. Foram, também, utilizados os herbicidas haloxyfop-methyl, fluazifopp-butyl + fomesafen, fluazifop-p-butyl, metolachlor, diclosulam ou chlorimuron-ethyl. Nos anos de cultivo do milho, foi utilizada a mistura dos herbicidas atrazine + metolachlor. Não foram aplicados herbicidas ao cultivo de milheto. As doses utilizadas seguiram as recomendações de cada produto (Rodrigues \& Almeida, 2005). O preparo convencional foi efetuado apenas na implantação (1991) e na renovação (2000) da pastagem. Nesse sistema de cultivo, as adubações foram feitas de acordo com as recomendações para as espécies, com base nos resultados de análise do solo e de produtividade esperada. Não foram utilizados herbicidas na pastagem.

Tabela 1. Histórico de culturas e total de calcário e de fertilizantes aplicados nos sistemas de cultivo em área experimental da Embrapa Cerrados, Planaltina, DF.

\begin{tabular}{|c|c|c|c|c|c|c|c|c|}
\hline \multirow[t]{2}{*}{ Sistema de cultivo } & \multicolumn{3}{|c|}{ Ciclos e culturas $^{(1)}$} & \multicolumn{5}{|c|}{ Adubação corretiva gradual } \\
\hline & 1991/1992-1994/1995 & 1995/1996-1998/1999 & $1999 / 2000-2003 / 2004$ & $\begin{array}{l}\text { Calcário } \\
\left(\mathrm{Mg} \mathrm{ha}^{-1}\right)\end{array}$ & $\begin{array}{c}\mathrm{N} \\
----\end{array}$ & $\begin{array}{r}\mathrm{P}_{2} \mathrm{O}_{5} \\
-(\mathrm{kg} \mathrm{ha} \\
\end{array}$ & $\mathrm{K}_{2} \mathrm{O}$ & $\begin{array}{c}\text { Gesso } \\
\left(\mathrm{Mg} \mathrm{ha}^{-1}\right)\end{array}$ \\
\hline ontínua & $\mathrm{S} / \mathrm{S} / \mathrm{M} / \mathrm{S}$ & $\mathrm{M} / \mathrm{S} / \mathrm{M} / \mathrm{S}$ & $\mathrm{S} / \mathrm{Mi} / \mathrm{S} / \mathrm{Mi} / \mathrm{S}$ & 7,5 & 235 & 1.123 & 1.037 & 2,8 \\
\hline Pastagem contínua & $\mathrm{A} / \mathrm{A} / \mathrm{A} / \mathrm{A}$ & $\mathrm{A} / \mathrm{A} / \mathrm{A} / \mathrm{A}$ & $\mathrm{A} / \mathrm{Bd} / \mathrm{Bd} / \mathrm{Bd} / \mathrm{Bd}$ & 6,8 & 80 & 254 & 204 & 0,0 \\
\hline
\end{tabular}

(1) A, capim-andropógon (Andropogon gayanus Kunth); Bd, capim-braquiária (Urochloa decumbens (Stapf) R. Webster); M, milho (Zea mays L.); Mi, milheto (Pennisetum americanum (L.) Leeke); S, soja (Glycine max (L.) Merr.). 
As amostras de solo foram coletadas na safra de 2003/2004, depois da colheita da soja e antes da primeira gradagem da área para o preparo da área de plantio. As coletas de solo foram realizadas entre outubro e novembro de 2004. Adotou-se amostragem sistemática por conglomerados, com coleta de quatro amostras compostas por quatro subamostras com diâmetro de $7,3 \mathrm{~cm}$. As coletas foram realizadas em linha no sentido da largura nas áreas, à profundidade entre 0 e $5 \mathrm{~cm}$. A distância entre os pontos de amostragem foi de $10 \mathrm{~m}$, tendo-se desconsiderado $5 \mathrm{~m}$ de bordadura.

Depois da secagem ao ar, as amostras de solo foram homogeneizadas. Foram utilizadas bandejas de plásticos de 19,5x19,5x6,5 cm numeradas, perfuradas e acrescidas de areia esterilizada, para se facilitar a drenagem da água e evitar o escoamento das amostras. Foram distribuídas, também, bandejas de controle com somente areia esterilizada, para o monitoramento de contaminação externa de sementes.

As amostras foram irrigadas diariamente, a partir de dezembro de 2004. O solo foi revolvido, depois de cinco dias sem irrigação, aos seis meses após a emergência e avaliação, para que novos fluxos de emergência pudessem ocorrer. A emergência de plântulas cessou ao final de aproximadamente oito meses de avaliação, tendo havido 16 levantamentos, com intervalos de aproximadamente 15 dias. $\mathrm{O}$ total de sementes foi obtido pela soma de todas as plântulas emergidas, em cada amostra, durante esse período. Foram coletadas amostras de plântulas, plantas adultas e plantas com flores e frutos, para confirmar a identificação botânica em herbário. Esse procedimento foi efetuado na Embrapa Recursos Genéticos e Biotecnologia e na Reserva Ecológica do IBGE. As amostras com flores e frutos foram depositadas no Herbário do IBGE.

As sementes que germinaram e morreram, antes de serem identificadas, constaram apenas da avaliação de densidade de sementes. Os dados foram expressos em sementes por metro quadrado. A densidade de sementes foi comparada pelo teste não-paramétrico de Wilcoxon $(\mathrm{p}=0,05)$, pelo SAS (SAS Institute, 2000). Avaliou-se também o índice de valor de importância (IVI) (MuellerDombois \& Ellenberg, 1974), de cada espécie, em todas as áreas, tendo-se somado o IVI de espécies nativas de cerrado e de invasoras, além do IVI de cada família, para cada área avaliada. O IVI foi obtido pela soma da densidade relativa com a frequiência relativa, tendo-se considerado as espécies resultantes do conjunto de amostras de cada área. Além disso, avaliou-se a percentagem de espécies nativas de cerrado e de invasoras. Embora as espécies nativas, encontradas em área de pastagem ou de lavoura, sejam consideradas invasoras nessas áreas, elas foram separadas das demais espécies por não serem consideradas espécies daninhas verdadeiras (Zimdhal, 1993).

Calculou-se também o índice de diversidade de Shannon (H') e de Simpson (D) (Magurran, 1988), pelo programa MVSP 3.1.

$\mathrm{H}^{\prime}=-\sum_{\mathrm{i}=1}^{\mathrm{n}} \mathrm{pi} * \mathrm{Ln} *(\mathrm{pi})$ em que,

Ln é o logaritmo neperiano; $\mathrm{pi}=$ ni/N; ni é o número de indivíduos amostrados da espécie $\mathrm{i}$; $\mathrm{N}$ é o número total de indivíduos amostrados.

$\mathrm{D}=\Sigma\left[\right.$ ni*(ni-1) / $\left.\mathrm{N}^{*}(\mathrm{~N}-1)\right]$ em que, ni é o número de indivíduos da espécie i; $\mathrm{N}$ é o número total de indivíduos.

\section{Resultados e Discussão}

Não ocorreu contaminação por sementes externas ao experimento, de acordo com os resultados das bandejas-controle. Nos resultados das amostras, observou-se que a maior densidade de sementes $(\mathrm{p}=0,05)$ ocorreu na área de lavoura (Tabela 2).

A área de cerrado queimada não apresentou monocotiledôneas, enquanto na área de pastagem essas espécies foram as que apresentaram maior IVI (Tabela 3). Na área de cerrado não queimada e na de

Tabela 2. Densidade absoluta de sementes, percentagem de espécies nativas e de invasoras e índice de diversidade de Shannon e de Simpson, do banco de sementes de solo sob cerrado queimado e não queimado, pastagem e lavoura com preparo convencional do solo, em área experimental da Embrapa Cerrados, Planaltina, DF, à profundidade de 0 a $5 \mathrm{~cm}$.

\begin{tabular}{|c|c|c|c|c|c|}
\hline \multirow[t]{2}{*}{ Área } & \multirow{2}{*}{$\begin{array}{c}\text { Densidade de } \\
\text { sementes }\left(\mathrm{n}^{\mathrm{o}} \mathrm{m}^{-2}\right)\end{array}$} & \multirow{2}{*}{$\begin{array}{c}\text { Espécies } \\
\text { nativas }(\%)\end{array}$} & \multirow{2}{*}{$\begin{array}{c}\text { Espécies } \\
\text { invasoras }(\%)\end{array}$} & \multicolumn{2}{|c|}{ Índice de diversidade } \\
\hline & & & & Shannon (Ln) & Simpson (Ln) \\
\hline Cerrado queimado & $134 \mathrm{a}$ & 75,0 & 25,0 & 1,27 & 0,69 \\
\hline Cerrado não queimado & $553 \mathrm{ab}$ & 41,7 & 58,3 & 2,06 & 0,82 \\
\hline Pastagem & $762 b$ & 61,5 & 38,5 & 2,05 & 0,80 \\
\hline Lavoura & $18.591 \mathrm{c}$ & 90,0 & 10,0 & 1,94 & 0,79 \\
\hline
\end{tabular}


lavoura, houve praticamente a mesma importância entre monocotiledôneas e dicotiledôneas.

O número de famílias na área de cerrado queimada foi o menor, entre todas as áreas avaliadas; Asteraceae e Rubiaceae foram as únicas famílias encontradas, enquanto as outras áreas apresentaram o mesmo número de famílias. Na área de cerrado queimada, a família Rubiaceae foi a mais importante, ao passo que nas outras áreas foi a Poaceae. Entretanto, essa última família não ocorreu na área de cerrado queimada, embora tenha sido a família com o maior número de espécies nas áreas em que ocorreu. A segunda família com maior número de espécies, na área de lavoura, foi a Asteraceae.

A área de lavoura apresentou o maior número de espécies, e o menor número ocorreu na área de cerrado queimada. Sabicea cf. brasiliensis foi a única espécie que ocorreu em todas as áreas, enquanto Ageratum conyzoides e Eleusine indica ocorreram em três das quatro áreas (Tabela 4). Quatro espécies nativas de cerrado estavam presentes apenas na área de cerrado sem queimada, enquanto Digitaria fuscescens, espécie também considerada nativa, ocorreu apenas na área de pastagem. Algumas das espécies invasoras ocorreram apenas em uma das áreas avaliadas. Conyza canadensis e Spermacoce cf. verticilata ocorreram apenas na área de cerrado queimada; Andropogon gayanus e Melinis minutiflora ocorreram apenas na área de cerrado sem queimada. Brachiaria decumbens, Portulaca oleracea e Tridax procumbens ocorreram apenas na área de pastagem, enquanto 11 espécies invasoras ocorreram apenas na área de lavoura. Outras espécies ocorreram apenas nas áreas cultivadas: Cenchrus echinatus, Digitaria cf. horizontalis e Solanum americanum, inclusive Eragrostis rufescens, espécie considerada nativa de cerrado (Mendonça et al., 1998). Houve, também, redução mais pronunciada dos índices de diversidade (Shannon e Simpson) na área de cerrado queimada (Tabela 2). As espécies com o maior IVI nas áreas foram: A. conyzoides (lavoura), B. decumbens (pastagem), M. minutiflora (cerrado sensu stricto) e $S$. cf. brasiliensis (Tabela 4). A espécie nativa $S$. cf. brasiliensis foi a segunda mais importante na área de pastagem, assim como Schwenckia americana, outra espécie nativa, na área de cerrado não queimada (Mendonça et al., 1998). A percentagem de espécies nativas decresceu, e a de espécies invasoras aumentou na seguinte ordem: cerrado não queimado, pastagem, cerrado queimado e lavoura (Tabela 2). A área de cerrado não queimada foi a que apresentou maior IVI de espécies nativas, seguida pela de cerrado queimada e pela pastagem e depois pela lavoura (Tabela 4). Apesar de ser a área com maior IVI de espécies nativas, a área de cerrado apresentou aproximadamente a metade do total de IVI correspondente às espécies invasoras.

O efeito da queimada sobre a densidade de sementes, em área de cerrado, provavelmente foi influenciado pelo período de permanência sob a ação do fogo e pela

Tabela 3. Índice de valor de importância (IVI) e número de espécies de famílias, em banco de sementes de solo sob cerrado queimado e não queimado, pastagem e lavoura com preparo convencional do solo, em área experimental da Embrapa Cerrados, Planaltina, DF, à profundidade de $0 \mathrm{a} 5 \mathrm{~cm}$.

\begin{tabular}{|c|c|c|c|c|c|c|c|c|}
\hline \multirow[t]{2}{*}{ Família } & \multicolumn{2}{|c|}{ Cerrado queimado } & \multicolumn{2}{|c|}{ Cerrado não queimado } & \multicolumn{2}{|c|}{ Pastagem } & \multicolumn{2}{|c|}{ Lavoura } \\
\hline & IVI $(\%)$ & № de espécies & IVI (\%) & № de espécies & IVI $(\%)$ & № de espécies & IVI $(\%)$ & № de espécies \\
\hline & \multicolumn{8}{|c|}{ Magnoliopsida (dicotiledônea) } \\
\hline Amaranthaceae & $-^{(1)}$ & - & - & - & - & - & 7 & 1 \\
\hline Asteraceae & 83 & 2 & 19 & 1 & 18 & 2 & 63 & 6 \\
\hline Lamiaceae & - & - & 10 & 1 & - & - & - & - \\
\hline Portulacaceae & - & - & - & - & 8 & 1 & - & - \\
\hline Rubiaceae & 117 & 2 & 32 & 2 & 26 & 1 & 11 & 3 \\
\hline Solanaceae & - & - & 29 & 1 & 23 & 2 & 20 & 2 \\
\hline Não identificada & - & - & 10 & 1 & - & - & - & - \\
\hline Subtotal & 200 & 4 & 99 & 6 & 74 & 6 & 101 & 12 \\
\hline \multirow[t]{2}{*}{ № de famílias } & \multicolumn{2}{|r|}{2} & \multicolumn{2}{|r|}{4} & \multicolumn{2}{|c|}{4} & \multicolumn{2}{|c|}{4} \\
\hline & \multicolumn{8}{|c|}{ Liliopsida (monocotiledônea) } \\
\hline Commelinaceae & - & - & - & - & - & - & 6 & 1 \\
\hline Cyperaceae & - & - & 19 & 2 & 8 & 1 & - & - \\
\hline Poaceae & - & - & 83 & 4 & 118 & 6 & 94 & 7 \\
\hline Subtotal & 0 & - & 102 & 6 & 126 & 7 & 100 & 8 \\
\hline № de famílias & \multicolumn{2}{|r|}{0} & \multicolumn{2}{|r|}{2} & \multicolumn{2}{|c|}{2} & \multicolumn{2}{|c|}{2} \\
\hline Total & 200 & 4 & 200 & 12 & 200 & 13 & 200 & 20 \\
\hline № total de famílias & \multicolumn{2}{|r|}{2} & \multicolumn{2}{|r|}{6} & \multicolumn{2}{|c|}{6} & \multicolumn{2}{|c|}{6} \\
\hline
\end{tabular}

${ }^{(1)}$ Família não encontrada na área. 
temperatura máxima atingida (Mistry, 1998). De acordo com Rheinheimer et al. (2003), a temperatura pode chegar a $70^{\circ} \mathrm{C}$ à profundidade de $0 \mathrm{a} 2 \mathrm{~cm}$, em queimada com duração de 4 min. No entanto, em razão da ocorrência acidental da queimada, não foi avaliada a temperatura máxima atingida ou o seu tempo de duração. O número de famílias na área de cerrado queimada foi menor do que na área de cerrado não queimada, e poderia ser citado como efeito da queimada e da ausência das famílias Poaceae e Cyperaceae na área queimada. De acordo com Cardoso et al. (2000), a queimada reduziu a frequiência das gramíneas predominantes e condicionou aumento no número de espécies dicotiledôneas e ciperáceas, em savana gramíneo-lenhosa no Pantanal. Os mesmos autores observaram que a queimada proporcionou maior número de espécies do que a área sem queimada, diferentemente dos resultados observados neste trabalho. A ausência de $M$. minutiflora, no banco de sementes da área queimada de cerrado, pode indicar que a queimada tenha inviabilizado as sementes (Kissmann \& Groth, 1997). Entretanto, esse efeito pode ser temporário, já que Martins et al. (2004) verificaram que houve aumento da área de cobertura dessa espécie, em três anos depois da ocorrência de queimada, em cascalheira localizada no Parque Nacional de Brasília. As espécies gramíneas nativas de cerrado se

Tabela 4. Índice de valor de importância (IVI) das espécies encontradas num banco de sementes de solo sob cerrado queimado e não queimado, pastagem e lavoura com preparo convencional do solo, em área experimental da Embrapa Cerrados, Planaltina, DF, à profundidade de 0 a 5 cm.

\begin{tabular}{|c|c|c|c|c|c|}
\hline Espécie & Família & Cerrado queimado & Cerrado não queimado & Pastagem & Lavoura \\
\hline \multicolumn{6}{|c|}{ Nativas do Cerrado } \\
\hline Sabicea cf. brasiliensis Wernham & Rubiaceae & 69,4 & 22,2 & 25,5 & 2,1 \\
\hline Morfotipo 1 & Cyperaceae & $-(1)$ & 9,5 & 7,6 & - \\
\hline Schwenckia americana D. Royen ex L. & Solanaceae & - & 28,8 & 7,6 & - \\
\hline Eragrostis rufescens Roem. \& Schult. & Poaceae & - & - & 7,6 & 4,9 \\
\hline Bulbostylis capillaris (L.) Kunth ex C.B. Clarke & Cyperaceae & - & 9,5 & - & - \\
\hline Morfotipo 2 & Não identificada & - & 9,5 & - & - \\
\hline Hyptis sp. & Lamiaceae & - & 9,5 & - & - \\
\hline Paspalum multicaule Poir. & Poaceae & - & 9,5 & - & - \\
\hline Digitaria fuscescens (J. Presl.) Henr. & Poaceae & - & - & 12,9 & - \\
\hline Subtotal & & 69,4 & 98,4 & 61,3 & 7,0 \\
\hline \multicolumn{6}{|c|}{ Invasoras } \\
\hline Ageratum conyzoides $\mathrm{L}$. & Asteraceae & 36,1 & - & 10,3 & 39,9 \\
\hline Eleusine indica (L.) Gaertn. & Poaceae & - & 9,5 & 23,2 & 20,5 \\
\hline cf. Achyrocline satureoides DC. & Asteraceae & - & 19,0 & - & 2,1 \\
\hline Richardia brasiliensis Gomes & Rubiaceae & - & 9,5 & - & 7,0 \\
\hline Cenchrus echinatus L. & Poaceae & - & - & 7,6 & 11,0 \\
\hline Digitaria cf. horizontalis Schumach. & Poaceae & - & - & 7,6 & 36,6 \\
\hline Solanum americanum Mill. & Solanaceae & - & - & 15,3 & 14,8 \\
\hline Conyza canadensis (L.) Cronq. & Asteraceae & 47,2 & - & - & - \\
\hline Spermacoce cf. verticilata L. & Rubiaceae & 47,2 & - & - & - \\
\hline Andropogon gayanus Kunth & Poaceae & - & 19,0 & - & - \\
\hline Melinis minutiflora P. Beauv. & Poaceae & - & 44,8 & - & - \\
\hline Portulaca oleracea L. & Portulacaceae & - & - & 7,6 & - \\
\hline Tridax procumbens L. & Asteraceae & - & - & 7,6 & - \\
\hline Urochloa decumbens (Stapf) R.D.Webster & Poaceae & - & - & 59,5 & - \\
\hline Acanthospermum australe (Loefl.) Kuntze & Asteraceae & - & - & - & 2,3 \\
\hline Alternanthera tenella Colla & Amaranthaceae & - & - & - & 6,9 \\
\hline Blainvillea rhomboidea Cass. & Asteraceae & - & - & - & 6,5 \\
\hline Centratherum cf. camporum (Hassl.) Malme & Asteraceae & - & - & - & 9,6 \\
\hline Commelina benghalensis L. & Commelinaceae & - & - & - & 5,6 \\
\hline Digitaria ciliaris (Retz.) Koeler & Poaceae & - & - & - & 11,8 \\
\hline Emilia sonchifolia DC. & Asteraceae & - & - & - & 2,3 \\
\hline Nicandra physaloides Gaertn. & Solanaceae & - & - & - & 4,9 \\
\hline Pennisetum spp. & Poaceae & - & - & - & 7,1 \\
\hline Rhynchelitrum repens (Willd.) C.E. Hubb. & Poaceae & - & - & - & 2,1 \\
\hline Spermacoce cf. capitata ex DC. & Rubiaceae & - & - & - & 2,1 \\
\hline Subtotal & & 130,6 & 101,6 & 138,7 & 193,0 \\
\hline Total geral & & 200,0 & 200,0 & 200,0 & 200,0 \\
\hline
\end{tabular}

${ }^{(1)}$ Espécie não encontrada na área. 
comportariam como estrategista $\mathrm{R}$ (rápido crescimento populacional, típico de ambientes variáveis), com a ocorrência de queimada, e como estrategista K (lento crescimento populacional, típico de ambientes estáveis), quando há longos períodos sem queimada (Mistry, 1998). Os índices de diversidade (Shannon e Simpson) também indicaram que a queimada proporcionou menor diversidade no banco de sementes. Os valores encontrados, em ambas as áreas, podem ser considerados baixos se comparados com os valores observados para o estrato arbóreo de cerrado, o que caracteriza a perturbação das áreas, mesmo no caso da área não queimada. Rossi et al. (1998) encontraram valores entre 3,11 e 3,71, para o índice de Shannon, em 11 localidades de cerrado sensu stricto no Brasil Central. Além disso, deve-se considerar que, no levantamento do estrato arbóreo, também são contados os indivíduos originários de rebrotos.

O maior banco de sementes observado no cultivo de lavoura, provavelmente, está relacionado ao maior revolvimento do solo nessa área do que na de pastagem (Ikeda et al., 2007a). Acredita-se que a família Asteraceae tenha sido mais importante na área de lavoura, em razão da maior luminosidade ali existente do que nas outras áreas, já que esse fator parece ser importante para a germinação das sementes dessa família (Ikeda et al., 2007b). A família Poaceae foi a mais importante nas áreas cultivadas e de cerrado sem queimada, provavelmente em razão da capacidade de estabelecimento de grande parte das espécies a ela pertencentes (Kissmann \& Groth, 1997). A família Rubiaceae foi a mais importante na área de cerrado queimada, principalmente pela presença de S. cf. brasiliensis. A queimada, nesse caso, aparentemente afeta menos essa espécie. A. conyzoides é uma espécie com elevada produção de sementes e dispersão pelo vento (Kissmann \& Groth, 1999), o que explica sua presença em todas as áreas avaliadas. É uma espécie da família Asteraceae que apresenta preferência por áreas com maior luminosidade (Ikeda et al., 2007a). A pastagem avaliada apresentava $B$. decumbens como forrageira, o que justifica o elevado IVI dessa espécie na área. M. minutiflora, principal espécie na área de cerrado não queimada, é uma gramínea exótica de origem africana, com reprodução por sementes (Kissmann \& Groth, 1997), que tem proporcionado maior impacto sobre a flora do Parque Nacional de Brasília (Martins et al., 2004). S. cf. brasiliensis foi a segunda mais importante na área de pastagem, assim como S. americana na área de cerrado, o que indica a capacidade de propagação dessas espécies nativas (Mendonça et al., 1998). As espécies invasoras, geralmente, apresentam elevada produção de sementes (Zimdhal, 1993), enquanto a maior parte das espécies nativas de cerrado aparenta ter maior dificuldade para o desenvolvimento em áreas com maior perturbação do solo, como no caso da área de lavoura. Essa seria a justificativa para que as espécies nativas sejam consideradas as de manejo mais complexo, nas áreas de pastagem, onde acabam atuando como invasoras (Pott et al., 2006). A facilidade de dispersão e de desenvolvimento, além da capacidade de competição das espécies consideradas invasoras (Zimdhal, 1993) e a proximidade da área de cerrado com os sistemas agrícolas, explicariam a importância dessas espécies nas áreas de cerrado, mesmo tendo áreas de pastagem delimitando as áreas de cerrado sensu stricto.

\section{Conclusões}

1. O cultivo mais intenso (lavoura com preparo convencional do solo) e a queimada são condições que favorecem o banco de sementes de espécies invasoras, em detrimento de espécies nativas.

2. O cultivo com lavoura causa maior perturbação nas áreas do que a queimada.

\section{Agradecimentos}

À Embrapa Cerrados e ao Institut de Recherche pour le Développement (IRD), Ministère de Affaires Étrangères (MAE), pelo apoio no desenvolvimento deste trabalho.

\section{Referências}

AGUIAR, L.M. de S.; MACHADO, R.B.; MARINHO-FILHO, J. A diversidade biológica do Cerrado. In: AGUIAR, L.M. de; CAMARGO, A.J.A. (Org.). Cerrado: ecologia e caracterização. Planaltina: Embrapa Cerrados; Brasília: Embrapa Informação Tecnológica, 2004. p.17-40.

BALL, D.A.; MILLER, S.D. A comparison of techniques for estimation of arable soil seedbanks and their relationship to weed flora. Weed Research, v.29, p.365-373, 1989.

CARDOSO, E.L.; CRISPIM, S.M.A.; RODRIGUES, C.A.G.; BARIONI JÚNIOR, W. Composição e dinâmica da biomassa aérea 
após a queima em savana gramíneo-lenhosa no Pantanal. Pesquisa Agropecuária Brasileira, v.35, p.2309-2316, 2000.

FELFILI, J.M.; SILVA JÚNIOR, M.C. (Org.). Biogeografia do bioma Cerrado: estudo fitofisionômico da Chapada do Espigão Mestre do São Francisco. Brasília: Unb, 2001. 152p.

HERINGER, I.; JACQUES, A.V.A. Adaptação das plantas ao fogo: enfoque na transição floresta-campo. Ciência Rural, v.31, p.10851090, 2001.

IKEDA, F.S.; MITJA, D.; CARMONA, R.; VILELA, L. Caracterização florística de bancos de sementes em sistemas de cultivo lavoura-pastagem. Planta Daninha, v.25, p.735-745, 2007a.

IKEDA, F.S.; MITJA, D.; VILELA, L.; CARMONA, R. Bancos de sementes no solo em sistemas de cultivo lavoura-pastagem. Pesquisa Agropecuária Brasileira, v.42, p.1545-1551, $2007 \mathrm{~b}$.

KISSMANN, K.G.; GROTH, D. Plantas infestantes e nocivas. 2.ed. São Paulo: BASF, 1997. Tomo I. 824p.

KISSMANN, K.G.; GROTH, D. Plantas infestantes e nocivas. 2.ed. São Paulo: BASF, 1999. Tomo II. 978p.

MAGURRAN, A.E. Ecological diversity and its measurement. Londres: Princeton University Press, 1988. 192p.

MARTINS, C.R.; LEITE, L.L.; HARIDASAN, M. Capim-gordura (Melinis minutiflora P. Beauv.), uma gramínea exótica que compromete a recuperação de áreas degradadas em unidades de conservação. Revista Árvore, v.28, p.739-747, 2004.

MEDEIROS, M.B.; MIRANDA, H.S. Mortalidade pós-fogo em espécies lenhosas de campo-sujo submetido a três queimadas prescritas anuais. Acta Botanica Brasilica, v.19, p.493-500, 2005.

MENDONÇA, R.C.; FELFILI, J.M.; WALTER, B.M.T.; SILVA JÚNIOR, M.C.; REZENDE, A.V.; FILGUEIRAS, T.S.; NOGUEIRA, P.E. Flora vascular do Cerrado. In: SANO, S.M.; ALMEIDA, S.P. Cerrado: ambiente e flora. Planaltina: EmbrapaCPAC, 1998. p.289-556.

MISTRY, J. Fire in the Cerrado (savannas) of Brazil: an ecological review. Progress in Physical Geography, v.22, p.425-448, 1998.

MOONEN, A.C.; BÀRBERI, P. Size and composition of the weed seedbank after 7 years of different cover-crop-maize management systems. Weed Research, v.44, p.163-177, 2004.
MUELLER-DOMBOIS, D.; ELLENBERG, H. Aims and methods of vegetation ecology. New York: John Wiley \& Sons, 1974. 570p. MURPHY, S.D.; CLEMENTS, D.R.; BELAOUSSOFF, S.; KEVAN, P.G.; SWANTON, C.J. Promotion of weed species diversity and reduction of weed seedbanks with conservation tillage and crop rotation. Weed Science, v.54, p.69-77, 2006.

POTT, A.; POTT, V.J.; SOUZA, T.W. Plantas daninhas de pastagem na região dos Cerrados. Campo Grande: Embrapa Gado de Corte, 2006. 336p.

RHEINHEIMER, D.S.; SANTOS, J.C.P.; FERNANDES, V.B.B.; MAFRA, A.L.; ALMEIDA, J.A. Modificações nos atributos químicos de solo sob campo nativo submetido à queima. Ciência Rural, v.33, p.49-55, 2003.

RODRIGUES, B.N.; ALMEIDA, F.S. Guia de herbicidas. 5.ed. Londrina: Os Autores, 2005. 592p.

ROSSI, C.V.; SILVA JÚNIOR, M.C.; SANTOS, C.E.N. Fitossociologia do estrato arbóreo de Cerrado (sensu stricto) no Parque Ecológico Norte, Brasília-DF. Boletim do Herbário Ezechias Heringer, v.2, p.49-56, 1998.

SANO, E.E.; BEZERRA, H. da S.; BARCELLOS, A. de O.; ROSA, R. Metodologias para mapeamento de pastagens degradadas no Cerrado. Planaltina: Embrapa Cerrados, 2002. 22p. (Embrapa Cerrados. Boletim de Pesquisa e Desenvolvimento, 70).

SANO, E.E.; JESUS, E.T.; BEZERRA, H.S. Uso de um sistema de informações geográficas para quantificação de áreas remanescentes do Cerrado. Planaltina: Embrapa Cerrados, 2001. 4p. (Embrapa Cerrados. Comunicado Técnico, 62).

SAS INSTITUTE. SAS/STAT: user's guide: version 8. Cary: Sas Institute, 2000.

SASSAKI, R.M.; RONDON, J.N.; ZAIDAN, L.B.P.; FELIPPE, G.M. Number of buried seeds and seedlings emergence in cerradão, cerrado and gallery forest soils at Pedregulho, Itirapina (SP), Brazil. Revista Brasileira de Botânica, v.22, p.147-152, 1999.

SOSNOSKIE, L.M.; HERMS, C.P.; CARDINA, J. Weed seedbank community composition in a 35-yr-old tillage and rotation experiment. Weed Science, v.54, p.263-273, 2006.

ZIMDHAL, R.L. Fundamentals of weed science. California: Academic Press, 1993. 450p.

Recebido em 10 de janeiro de 2008 e aprovado em 3 de junho de 2008 